\title{
Surface Roughness and Density Effects in Thermal Elastohydrodynamic Lubrication Point Contacts
}

\author{
Samuel Macharia Karimi ${ }^{1, *}$, Mathew Ngugi Kinyanjui ${ }^{2}$, Mark Kimathi ${ }^{3}$ \\ ${ }^{1}$ Institute of Basic Sciences, Technology and Innovation, Pan African University, Nairobi, Kenya \\ ${ }^{2}$ Department of Pure and Applied Mathematics, Jomo Kenyatta University of Agriculture and Technology, Nairobi, Kenya \\ ${ }^{3}$ Department of Mathematics, Statistics and Actuarial Science, Machakos University, Machakos, Kenya
}

Email address:

karimimacharia@gmail.com (S. M. Karimi)

${ }^{*}$ Corresponding author

\section{To cite this article:}

Samuel Macharia Karimi, Mathew Ngugi Kinyanjui, Mark Kimathi. Surface Roughness and Density Effects in Thermal Elastohydrodynamic Lubrication Point Contacts. American Journal of Mathematical and Computer Modelling. Vol. 5, No. 3, 2020, pp. 89-96. doi: 10.11648/j.ajmcm.20200503.15

Received: August 28, 2020; Accepted: September 10, 2020; Published: September 19, 2020

\begin{abstract}
Elastohydrodynamic lubrication is a type of lubrication which most machine elements such as bearings operate. Density changes, thermal and surface roughness effects are also key factors in bearings, working under heavy loads and high speeds. Previous research has focused on smooth surfaces where density and thermal effects have been neglected. The present study intends to model the effect of surface roughness and density on thermal elastohydrodynamic lubrication for sliding-rolling bearing using non-Newtonian lubricant. The surface roughness is incorporated into the film thickness equation while the non-Newtonian nature of the lubricant is incorporated into the Reynolds and energy equation by using the Eyring model. The changes in compressibility of the lubricant is given by the lubricant's density equation. The energy equation is solved simultaneously with the Reynolds-Eyring equation, film thickness, density and viscosity of lubricant equations. The equations are then discretized using the finite difference numerical method and are solved simultaneously in Matlab together with their boundary conditions. It is noted that an increase in surface roughness results to a reduction in the film thickness and an increase in both temperature and pressure. Increase in temperature lowers the density of the lubricant while increase in pressure leads to an increase in density. It is also noted that an increase in the density of the lubricant leads to an increase in the film thickness. The temperature profile shows that as the load in the bearing is increased, the temperature of the lubricant also increases.
\end{abstract}

Keywords: Density, Elastohydrodynamic, Surface Roughness, Thermal

\section{Introduction}

Lubrication is an important aspect for modern machine so that they can work efficiently, reduce friction and wear. Elastohydrodynamic lubrication (EHL) is a type of lubrication in tribology where high pressure in the contact region causes deformations. Thermal elastohydrodynamic lubrication (TEHL) also incorporates the effect of temperature on the lubricant and bearing surface because of friction that is generated by the surfaces in contact. Most machine components operate under elastohydrodynamic lubrication hence the design and analysis of how various parameters affect the bearing long life is important in sliding-rolling bearings.

The numerical work of electrohydrodynamic lubrication was established by the work of [1] together with the lab experimental work of the research [2]. Most research in elastohydrodynamic lubrication is based on this work including sliding-rolling conditions [3]. Thermal and non-Newtonian nature of the lubricant play an important role in elastohydrodynamic study hence they cannot be neglected. The research study noted that that an increase in temperature and non-Newtonian effects leads to a reduction in the film thickness $[4,5]$.

The effects of surface roughness have been a subject study in EHL since the 1970s. Bearing surfaces can longer be assumed smooth hence a lot of effort has gone into the study of rough surface contact in lubrication models. Surface roughness can be incorporated to the model by adding it to the film thickness [6]. The research [7] studied the effects of 
surface roughness in elastohydrodynamic problem. The surface roughness was determined by measuring the actual surface of the bearing contact. It was noted that the surface roughness significantly influenced the film thickness and pressure. The study [8] on surface roughness in pure sliding elastohydrodynamic lubrication with shear thinning lubricants noted that the surface roughness in the bearing caused sharp pressure peaks and reduced the minimum film thickness. The research study [9] investigated the effects of surface roughness on curve annular plates and concluded that the surface roughness increased the pressure in the bearing. Another research study [10], also investigated the effects of surface roughness on two rectangular plates and noted that surface roughness enhanced load carrying capacity, squeeze film time and pressure. The compressibility effect of lubricants plays an important role in lubrication theory. Under normal engineering conditions the density can be considered a constant. However, in rolling bearings, cams and gears the effects of high pressure and load results in changes in the lubricant volume which affects the density of the lubricant [11]. At this high load, the compressibility of the lubricant has a great influence on the film thickness hence various density models have been proposed [12]. The study [13] in their research of cylindrical rollers under heavy loads considered the density equation that varied with both pressure and density. The study [14] also considered the viscosity and density of the lubricant to be dependent on pressure and temperature. It is a known fact that temperature, pressure and film thickness play an important role in lubrication for highly loaded contacts. Under sliding conditions heat is generated due to the shearing which reduces the viscosity of the lubricant. Thus, investigations of how thermal effects affect the lubricant is crucial in TEHL study $[15,16]$. No one lubricant is Newtonian in nature. Past research assumed that lubricants are Newtonian which limited proper modelling of TEHL study. The non-Newtonian nature of lubricants has been subject to a lot of attention in lubrication study [17]. The non-Newtonian nature of the lubricants can be better incorporated into the Reynold and energy equation by using the Eyring model [18]. The study [4] noted that non-Newtonian and thermal effects lead to a reduction in the lubricant film thickness.

The Reynold and energy equations are nonlinear partial differential equations. Therefore, a numerical method has to be proposed to solve them. The research study [19] in elastohydrodynamic lubrication contacts with grease as a lubricant discretized their equations using the finite difference method. The research study [20] on determination of stiffness of pad thrust bearing used the finite difference method to discretize the two-dimensional equation and proved successful in solving the equations. The study [21] of surface roughness in thermohydrodynamic lubrication of a journal bearing discretized the energy and modified Reynolds equations using the finite difference method for a two-dimensional flow.

This research investigates the effects of density, load and surface roughness on thermal elastohydrodynamic lubrication.
To incorporate the density, load. Thermal and surface roughness for non-Newtonian lubricants for two-dimensional flow for sliding-rolling bearing, the Reynold-Eyring equation, energy equation, film thickness equation, rheology of lubricant equations ha to be solved. The finite difference numerical method is employed to solve these equations. The film thickness, pressure and temperature profiles are presented to show how various parameters and variables affect the lubricant.

\section{Geometry of the Model}

The contact geometry of the sliding-rolling bearing is represented by a rolling element that rolls over a rigid flat surface as shown in Figure 1. The bearing consists of a rolling circular element that slide or rolls over a flat surface. Due to high pressure and load, the surface and bearing deforms.

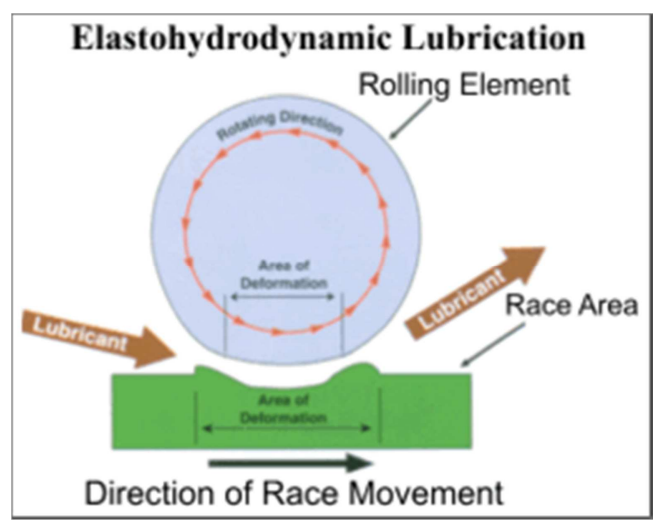

Figure 1. Geometry before deformation.

\section{Mathematical Equations}

The thermal elastohydrodynamic equations governing the flow of the lubricant include the Reynold-Eyring equation, energy equation, film thickness equation, the viscosity and density equations for the rheology of the lubricant. The point contact equations for the model in two-dimensional flow for $\mathrm{x}$ and y coordinates are resented.

\subsection{Eyring Model}

The lubricant to be considered is non-Newtonian in nature. The Eyring non-Newtonian model is proposed in this study to incorporate the changes in the viscosity of the lubricant. The Eyring equation is given by

$$
\gamma=\frac{\tau_{0}}{\eta} \sinh \left(\frac{\tau}{\tau_{0}}\right)
$$

\subsection{Reynolds-Eyring Equation}

The traditional Reynolds equation assumes that the lubricant is Newtonian in nature. Therefore, the Reynolds equation has to be modified to incorporate the non-Newtonian nature of the lubricant. This is done by incorporating the Eyring equation (1) into the two-dimensional Reynolds equation. Thus, the Reynolds-Eyring equation is given by; 


$$
\frac{\partial}{\partial x}\left[\frac{\rho h^{3}}{12 \eta} \frac{\partial p}{\partial x} S(x)\right]+\frac{\partial}{\partial y}\left[\frac{\rho h^{3}}{12 \eta} \frac{\partial p}{\partial y} Q(y)\right]=\frac{U_{m}}{2} \frac{\partial(\rho h)}{\partial x}+\frac{V_{m}}{2} \frac{\partial(\rho h)}{\partial y}+\frac{\partial \rho h}{\partial t}
$$

Where

$$
\begin{gathered}
S(x)=\frac{3(\xi \cosh (\xi)-\sinh (\xi))}{\xi^{3}} \cosh \left[\frac{\tau_{m}}{\tau_{0}}\right] \\
Q(y)=\frac{3(\delta \cosh (\delta)-\sinh (\delta))}{\delta^{3}} \cosh \left[\frac{\tau_{m}}{\tau_{0}}\right] \\
\xi=\frac{h}{2 \tau_{0}} \frac{\partial p}{\partial x}, \delta=\frac{h}{2 \tau_{0}} \frac{\partial p}{\partial y}
\end{gathered}
$$

\subsection{Energy Equation}

The energy equation after incorporating the non-Newtonian nature of the lubricant given by equation (1) is given by

$$
\begin{gathered}
\rho C_{p}\left(\frac{\partial \theta}{\partial T}+u \frac{\partial \theta}{\partial x}+v \frac{\partial \theta}{\partial y}\right)=k\left(\frac{\partial^{2} \theta}{\partial x^{2}}+\frac{\partial^{2} \theta}{\partial y^{2}}\right)-\frac{\theta}{\rho} \frac{\partial \rho}{\partial \theta}\left(u \frac{\partial P}{\partial x}+v \frac{\partial p}{\partial y}\right)+\frac{\tau_{0}}{\eta}\left(\frac{\partial P}{\partial x} \frac{h}{2}+\tau_{m}\right) \sinh \left[\frac{1}{\tau_{0}}\left(\frac{\partial P}{\partial x} \frac{h}{2}+\tau_{m}\right)\right]+ \\
\frac{\tau_{0}}{\eta}\left(\frac{\partial P}{\partial y} \frac{h}{2}+\tau_{m}\right) \sinh \left[\frac{1}{\tau_{0}}\left(\frac{\partial P}{\partial y} \frac{h}{2}+\tau_{m}\right)\right]
\end{gathered}
$$

With

$$
\begin{aligned}
& u=U_{1}+\frac{h \tau_{0}}{2 \eta \xi}\left[\cosh \left[\frac{\tau_{m}}{\tau_{0}}+\xi\right]-\cosh \left[\frac{\tau_{m}}{\tau_{0}}-\xi\right]\right] \\
& v=V_{1}+\frac{h \tau_{0}}{2 \eta \delta}\left[\cosh \left[\frac{\tau_{m}}{\tau_{0}}+\delta\right]-\cosh \left[\frac{\tau_{m}}{\tau_{0}}-\delta\right]\right]
\end{aligned}
$$

\subsection{Film Thickness Equation}

The surface roughness $S R$ are incoperated in the film thickness. This equation is given by

$$
h(x, y)=h_{00}+\frac{x^{2}}{2 R}+\frac{y^{2}}{2 R}+S R(x, y)+\frac{2}{\pi E^{\prime}} \int_{-\infty}^{\infty} \int_{-\infty}^{\infty} \frac{p\left(x^{\prime}, y^{\prime}\right)}{\sqrt{\left(x-x^{\prime}\right)^{2}+\left(y-y^{\prime}\right)^{2}}} d x^{\prime} d y^{\prime}
$$

\subsection{Viscosity Equation}

The viscosity of the lubricant is affected by temperature and pressure. The relationship between temperature, pressure and density can be described by the equation [22]

$$
\eta=\eta_{0} e^{\ln \left(\eta_{0}\right)+9.67\left[-1+\left(\left(1+5.1 \times 10^{-9} p\right)^{Z}\left(\frac{\theta-138}{\theta_{0}-138}\right)^{-S}\right)\right]}
$$

\subsection{Density Equation}

The density of the lubricant is affected by temperature and pressure. The relationship between temperature, pressure and density can be described by the equation [22]

$$
\rho=\rho_{0}\left[1+\frac{0.6 \times 10^{-9} p}{1+1.7 \times 10^{-9} p}-0.00065\left(\theta-\theta_{0}\right)\right]
$$

\subsection{Dimensionless Parameters}

The equations governing the flow are non-dimensionalized using dimensionless parameters and variables given by

$$
P=\frac{p}{p_{h}}, \bar{\theta}=\frac{\theta}{\theta_{0}}, X=\frac{x}{a}, Y=\frac{y}{a}, \bar{\rho}=\frac{\rho}{\rho_{0}}, \bar{\eta}=\frac{\eta}{n_{0}}, H=\frac{h R}{a^{2}}, H_{00}=\frac{h_{00} R}{a^{2}}, T=\frac{t u}{2 a}, U=\frac{u_{m} \eta_{0}}{R E^{\prime}}, V=\frac{v_{m} \eta_{0}}{R E^{\prime}}, \mathbb{R}=\frac{s_{r} R}{a^{2}},
$$




\subsubsection{Reynold-Eyring Equation in Dimensionless Form}

Assuming that the rolling speed $\mathrm{U}_{\mathrm{m}}=\mathrm{V}_{\mathrm{m}}$ in the $\mathrm{x}$ and $\mathrm{y}$ direction is equal and applying the dimensionless variables, the Reynold-Eyring equation (2) is given by,

$$
\frac{\partial}{\partial X}\left[\frac{\bar{\rho} H^{3}}{\bar{\eta}} \frac{\partial P}{\partial X} S(X)\right]+\frac{\partial}{\partial Y}\left[\frac{\bar{\rho} H^{3}}{\bar{\eta}} \frac{\partial P}{\partial Y} Q(X)\right]=\lambda\left[\frac{\partial(\bar{\rho} H)}{\partial X}+\frac{\partial(\bar{\rho} H)}{\partial Y}+\frac{\partial \bar{\rho} H}{\partial T}\right]
$$

If $\beta=\frac{\bar{\rho} H^{3}}{\bar{\eta}}$, the Reynold-Eyring equation (7) becomes

$$
\frac{\partial}{\partial X}\left[\beta \frac{\partial P}{\partial X} S(X)\right]+\frac{\partial}{\partial Y}\left[\beta \frac{\partial P}{\partial Y} Q(X)\right]=\lambda\left[\frac{\partial(\bar{\rho} H)}{\partial X}+V_{m} \frac{\partial(\bar{\rho} H)}{\partial Y}+\frac{\partial \bar{\rho} H}{\partial T}\right]
$$

With

$$
\lambda=\frac{6 \eta_{0} U R^{2}}{p_{h} a^{3}}, \xi=\frac{H a p_{h}}{2 R \tau_{0}} \frac{\partial P}{\partial X}, \delta=\frac{H a p_{h}}{2 R \tau_{0}} \frac{\partial P}{\partial Y},
$$

\subsubsection{Dimensionless Energy Equation}

The energy equation (3) after non dimensionalization becomes

$$
\begin{gathered}
\bar{\rho} \rho_{0} C_{p}\left(\frac{\theta_{0} U_{m}}{2 a} \frac{\partial \bar{\theta}}{\partial T}+\frac{\theta_{0} E^{\prime} R}{a \eta_{0}}\left(U \frac{\partial \bar{\theta}}{\partial X}+V \frac{\partial \bar{\theta}}{\partial Y}\right)\right)=k \frac{\theta_{0}}{a^{2}}\left(\frac{\partial^{2} \bar{\theta}}{\partial X^{2}}+\frac{\partial^{2} \bar{\theta}}{\partial Y^{2}}\right)-\frac{\bar{\theta} p_{h} E^{\prime} R \rho_{0}}{a \eta_{0}} \frac{\partial \bar{\rho}}{\partial \bar{\theta}}\left(U \frac{\partial P}{\partial X}+V \frac{\partial P}{\partial Y}\right) \\
+\frac{\tau_{0}}{\bar{\eta} \eta_{0}}\left(\frac{\partial P}{\partial X} \frac{H a^{2}}{2 R}+\tau_{m}\right) \sinh \left[\frac{1}{\tau_{0}}\left(\frac{\partial P}{\partial X} \frac{H a^{2}}{2 R}+\tau_{m}\right)\right]+\frac{\tau_{0}}{\bar{\eta} \eta_{0}}\left(\frac{\partial P}{\partial Y} \frac{H a^{2}}{2 R}+\tau_{m}\right) \sinh \left[\frac{1}{\tau_{0}}\left(\frac{\partial P}{\partial Y} \frac{H a^{2}}{2 R}+\tau_{m}\right)\right]
\end{gathered}
$$

The dimensionless velocities in $\mathrm{x}$ and $\mathrm{y}$ direction are given respectively by

$$
\begin{gathered}
U=\frac{\eta_{0}}{R E^{\prime}}\left[U_{1}+\frac{H a^{2} \tau_{0}}{2 R \bar{\eta} \eta_{0} \xi}\left[\cosh \left(\frac{\tau_{m}}{\tau_{0}}+\frac{z \xi R}{H a^{2}}\right)-\cosh \left(\frac{\tau_{m}}{\tau_{0}}-\xi\right)\right]\right] \\
V=\frac{\eta_{0}}{R E^{\prime}}\left[V_{1}+\frac{H a^{2} \tau_{0}}{2 \bar{\eta} \eta_{0} \delta}\left[\cosh \left(\frac{\tau_{m}}{\tau_{0}}+\frac{z \delta R}{H a^{2}}\right)-\cosh \left(\frac{\tau_{m}}{\tau_{0}}-\delta\right)\right]\right]
\end{gathered}
$$

\subsubsection{Dimensionless Film Thickness Equation}

The geometry of the point contact bearing is given by film thickness equation (4) and in dimensionless form is given by

$$
H(x, y)=H_{00}+\frac{X^{2}}{2}+\frac{Y^{2}}{2}+S R(X, Y)+\frac{2}{\pi^{2}} \int_{-\infty}^{\infty} \int_{-\infty}^{\infty} \frac{P\left(X^{\prime}, Y^{\prime}\right)}{\sqrt{\left(X-X^{\prime}\right)^{2}+\left(Y-Y^{\prime}\right)^{2}}} d X^{\prime} d Y^{\prime}
$$

\subsubsection{Dimensionless Viscosity Equation}

The viscosity equation (5) in dimensionless form is given by

$$
\bar{\eta}=e^{\ln \left(\eta_{0}\right)+9.67\left[-1+\left(\left(1+5.1 \times 10^{-9} P p_{h}\right)^{Z}\left(\frac{\bar{\theta} \theta_{0}-138}{\theta_{0}-138}\right)^{-S}\right)\right]}
$$

\subsubsection{Dimensionless Density Equation}

The density equation (6) in dimensionless form is given by

$$
\bar{\rho}=\left[1+\frac{0.6 \times 10^{-9} P p_{h}}{1+1.7 \times 10^{-9} P p_{h}}-0.00065 \theta_{0}(\bar{\theta}-1)\right]
$$

\subsection{Boundary Conditions}

The Reynolds-Eyring equation is determined in the pressure and cavitation (cav) region. Hence the boundary conditions for pressure and temperature equations are given by

$$
\left.\begin{array}{c}
P\left(X_{\text {in }}, Y\right) \\
=0, P\left(X_{\text {end }}, Y\right) \\
=0
\end{array}\right) 0, P\left(X, Y_{\text {in }}\right)=0, P\left(X, Y_{\text {end }}\right)
$$

$$
\frac{\partial P}{\partial X_{c a v}}=\frac{\partial P}{\partial Y_{c a v}}=0, \bar{\theta}\left(X, Y_{\text {in }}\right)=\theta_{0}, \bar{\theta}\left(X, Y_{\text {end }}\right)=\theta_{0}
$$

\section{Numerical Solutions}

The Reynolds-Eyring and energy equations are non-linear differential equations hence the finite difference numerical method is used to solve them. The derivatives of the partial differential equations are derived by truncating the Taylors series expansion for three successive grid points. The finite difference method is employed to discretise all the equations governing the flow and the equations are solved simultaneously in Matlab.

The Reynold-Eyring equation after discretization is given by 


$$
\begin{gathered}
P_{i, j}=\left[\begin{array}{c}
\frac{1}{(d x)^{2}}\left(\beta_{i-\frac{1}{2}, j} P_{i-1, j} S_{i-\frac{1}{2}, j}+\beta_{i+\frac{1}{2}, j} P_{i+1} S_{i+\frac{1}{2}, j}\right)+\frac{1}{(d y)^{2}}\left(\beta_{i, j-\frac{1}{2}} P_{i, j-1} S_{i, j-\frac{1}{2}}+\beta_{i, j+\frac{1}{2}} P_{i, j+1} S_{i, j+\frac{1}{2}}\right) \\
-\frac{\lambda}{d x}\left(H_{i, j} \bar{\rho}_{i, j}-H_{i-1, j} \bar{\rho}_{i-1, j}\right)-\frac{\lambda}{d y}\left(H_{i, j} \bar{\rho}_{i, j}-H_{i, j-1} \bar{\rho}_{i, j-1}\right)-\frac{\lambda}{d t}\left(H_{i, j}^{k+1} \bar{\rho}_{i, j}^{k+1}-H_{i, j}^{k} \bar{\rho}_{i, j}^{k}\right)
\end{array}\right] \\
\quad \div\left[\frac{1}{(d x)^{2}}\left(\beta_{i-\frac{1}{2}, j} S_{i-\frac{1}{2}, j}+\beta_{i+\frac{1}{2}, j} S_{i+\frac{1}{2}, j}\right)+\frac{1}{(d y)^{2}}\left(\beta_{i, j-\frac{1}{2}} S_{i, j-\frac{1}{2}}+\beta_{i, j+\frac{1}{2}} S_{i, j+\frac{1}{2}}\right)\right]
\end{gathered}
$$

Energy equation after discretisation is given by

$$
\begin{gathered}
{\left[\bar{\rho} \rho_{0} C_{p}\left(\frac{\theta_{0} E^{\prime} R}{a \eta_{0}}\left(U_{i, j} \bar{\theta}_{i+1, j}+V_{i, j} \frac{\bar{\theta}_{i, j+1}}{d y}\right)\right)-\frac{2 k \theta_{0}}{a^{2}}\left(\frac{\bar{\theta}_{i+1, j}+\bar{\theta}_{i-1, j}}{(d x)^{2}}+\frac{\bar{\theta}_{i, j+1}+\bar{\theta}_{i, j-1}}{(d y)^{2}}\right)-\frac{\tau_{0}}{\bar{\eta}_{i, j} \eta_{0}}\left(\frac{P_{i+1 . j}-P_{i, j}}{d x} \frac{H_{i, j} a^{2}}{2 R}+\tau_{m}\right) \sinh \left[\frac { 1 } { \tau _ { 0 } } \left(\frac{P_{i+1 . j}-P_{i, j}}{d x} \frac{H_{i, j} a^{2}}{2 R}+\right.\right.\right.} \\
\left.\left.\left.\tau_{m}\right)\right]-\frac{\tau_{0}}{\bar{\eta}_{i, j} \eta_{0}}\left(\frac{P_{i, j+1}-P_{i, j}}{d y} \frac{H_{i, j} a^{2}}{2 R}+\tau_{m}\right) \sinh \left[\frac{1}{\tau_{0}}\left(\frac{P_{i, j+1}-P_{i, j}}{d y} \frac{H_{i, j} a^{2}}{2 R}+\tau_{m}\right)\right]\right] \div\left(\bar{\rho}_{i, j} \rho_{0} C_{p}\left(\frac{\theta_{0} E^{\prime} R}{a \eta_{0}}\left(U \frac{\partial \bar{\theta}}{\partial x}+V \frac{\partial \bar{\theta}}{\partial Y}\right)\right)-\frac{2 k \theta_{0}}{a^{2}}\left(\frac{1}{(d x)^{2}}+\frac{1}{(d y)^{2}}\right)+\right. \\
\left.\frac{p_{h} E^{\prime} R \rho_{0}}{a \eta_{0}} 0.00065 \theta_{0}\left(U_{i, j} \frac{P_{i+1 . j}-P_{i, j}}{d x}+V_{i, j} \frac{P_{i, j+1}-P_{i, j}}{d y}\right)\right)
\end{gathered}
$$

With

$$
\begin{aligned}
& U_{i, j}=\frac{\eta_{0}}{R E^{\prime}}\left[U_{1}+\frac{H_{i, j} a^{2} \tau_{0}}{2 \bar{\eta}_{i, j} \eta_{0} \delta}\left[\cosh \left(\frac{\tau_{m}}{\tau_{0}}-\xi+\frac{2 z \xi R}{H_{i, j} a^{2}}\right)-\cosh \left(\frac{\tau_{m}}{\tau_{0}}-\xi\right)\right]\right] \\
& V_{i, j}=\frac{\eta_{0}}{R E^{\prime}}\left[V_{1}+\frac{H_{i, j} a^{2} \tau_{0}}{2 \bar{\eta}_{i, j} \eta_{0} \delta}\left[\cosh \left(\frac{\tau_{m}}{\tau_{0}}-\delta+\frac{2 z \delta R}{H_{i, j} a^{2}}\right)-\cosh \left(\frac{\tau_{m}}{\tau_{0}}-\delta\right)\right]\right]
\end{aligned}
$$

Film thickness equation after discretisation is given by

$$
H_{i, j}=H_{00}+\frac{X_{i, j}^{2}}{2}+\frac{Y_{i, j}^{2}}{2}+S R_{i, j}+\frac{2}{\pi^{2}} \sum_{r=0}^{n_{x}-1} \sum_{s=0}^{n_{y}-1} K_{r, i, s, j} P_{r S}
$$

where

$$
\begin{gathered}
K_{r, i, s, j}=\left|X_{p}\right| \sinh ^{-1}\left(\frac{Y_{p}}{X_{p}}\right)+\left|Y_{p}\right| \sinh ^{-1}\left(\frac{X_{p}}{Y_{p}}\right)-\left|X_{m}\right| \sinh ^{-1}\left(\frac{Y_{p}}{X_{m}}\right)+\left|Y_{p}\right| \sinh ^{-1}\left(\frac{X_{m}}{Y_{p}}\right)-\left|X_{p}\right| \sinh ^{-1}\left(\frac{Y_{m}}{X_{p}}\right) \\
+\left|Y_{m}\right| \sinh ^{-1}\left(\frac{X_{p}}{Y_{m}}\right)+\left|X_{m}\right| \sinh ^{-1}\left(\frac{Y_{m}}{X_{m}}\right)+\left|Y_{m}\right| \sinh ^{-1}\left(\frac{X_{m}}{Y_{m}}\right) \\
X_{p}=X_{i}-X_{r}+\frac{d x}{2}, X_{m}=X_{i}-X_{r}-\frac{d x}{2}, Y_{p}=Y_{j}-Y_{s}+\frac{d y}{2}, Y_{m}=Y_{j}-Y_{s}-\frac{d y}{2}
\end{gathered}
$$

The viscosity equation after discretization is given by

$$
\bar{\eta}_{i, j}=e^{\ln \left(\eta_{0}\right)+9.67\left[-1+\left(\left(1+5.1 \times 10^{-9} P_{i, j} p_{h}\right)^{z}\left(\frac{\bar{\theta}_{i, j} \theta_{0}-138}{\theta_{0}-138}\right)^{-S}\right)\right]}
$$

The density equation after discretization is given by

$$
\bar{\rho}_{i, j}=\left[1+\frac{0.6 \times 10^{-9} P_{i, j} p_{h}}{1+1.7 \times 10^{-9} P_{i, j} p_{h}}-0.00065 \theta_{0}\left(\bar{\theta}_{i, j}-1\right)\right]
$$

\section{Results and Discussion}

A two-dimensional non-Newtonian model for the thermal electrohydrodynamic lubrication is proposed. The film thickness, temperature and pressure distribution along the $\mathrm{z}$ axis are considered to be a constant hence the bearing rolls along the $\mathrm{x}$ and $\mathrm{y}$ axis only. The profiles are represented along one bearing direction which is the x-axis. Density, surface roughness and load changes are investigated. The film thickness, pressure and temperature profiles are then presented and discussed. 


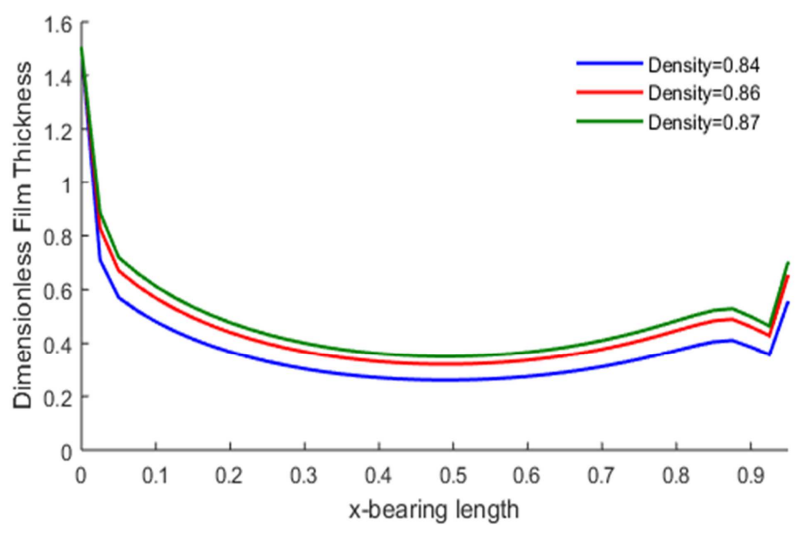

Figure 2. Effects of Density on Film Thickness.

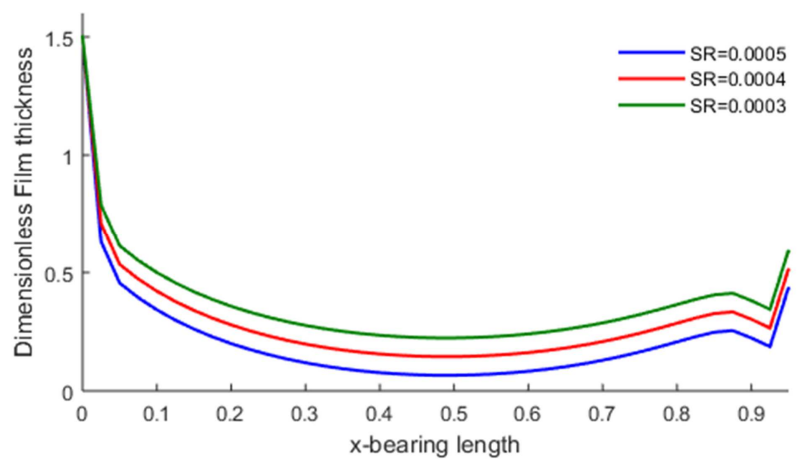

Figure 3. Effects of Surface Roughness on Film Thickness.

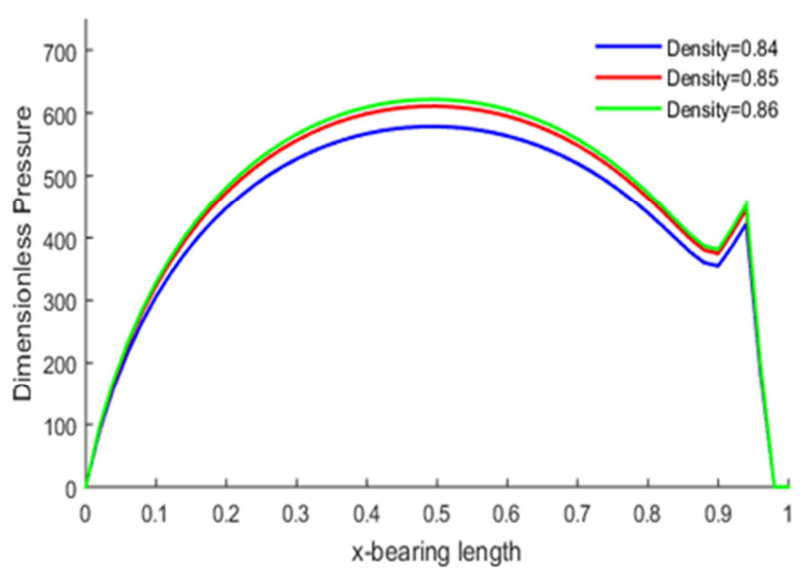

Figure 4. Effects of Pressure on Density.

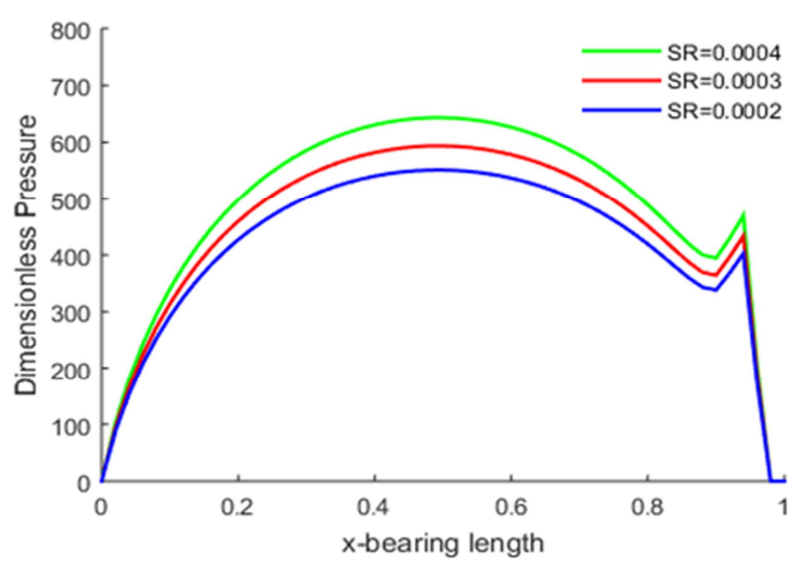

Figure 5. Effects of Surface Roughness on Pressure.

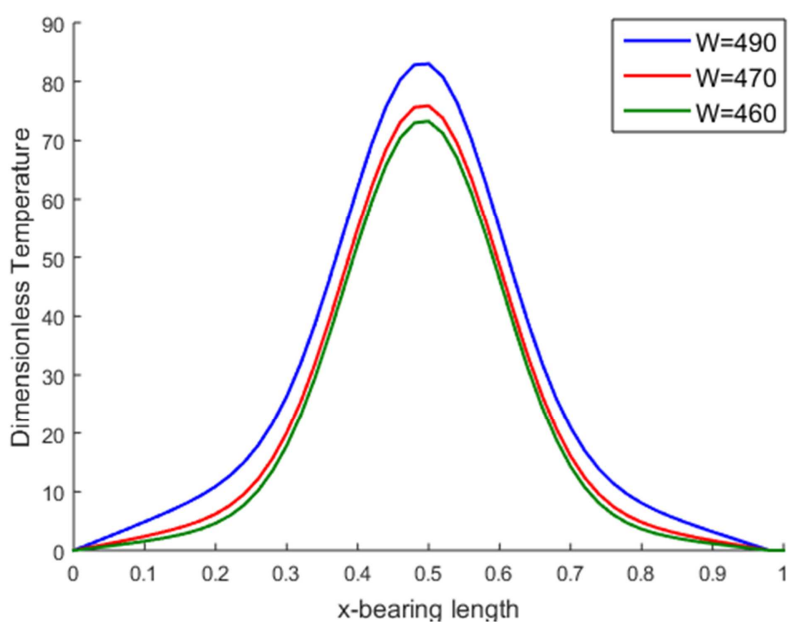

Figure 6. Effects of Load on Temperature.

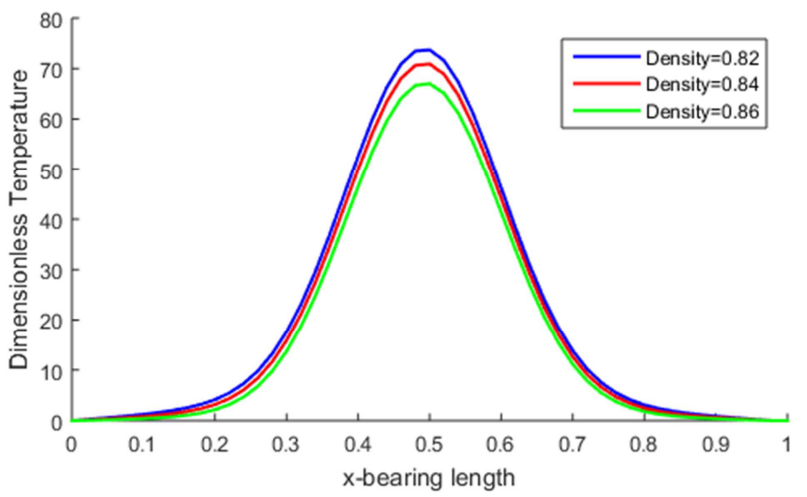

Figure 7. Effects of Temperature on Density.

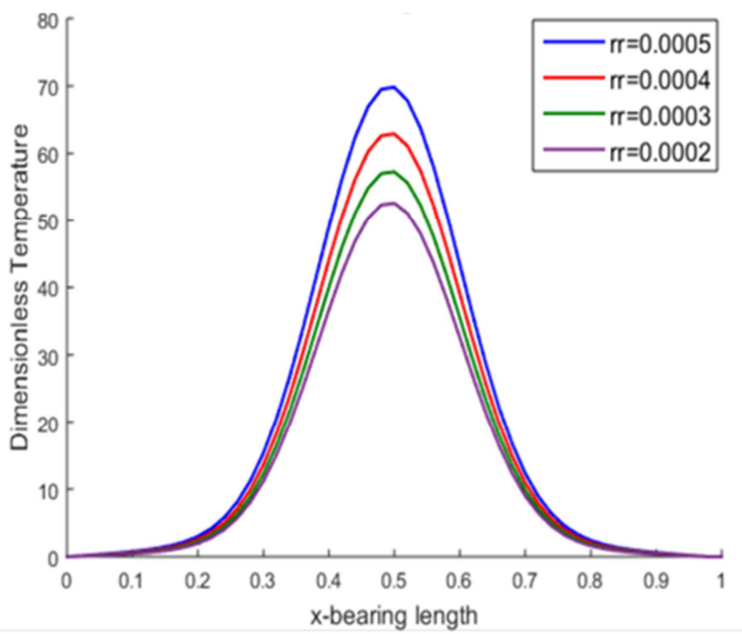

Figure 8. Effects of Surface Roughness on Temperature.

It is noted from Figure 2 that an increase in the density of the lubricant leads to an increase in height in the film thickness. This is due to the fact that an increase in density results to the lubricant becoming thicker hence an increase in the viscosity. Figure 3 shows that an increase in the surface roughness results to a decrease in the film thickness. This reduction in the film thickness is due to the negative pressure gradient due to the surface roughness as compared when the bearing has a smooth surface. The reduction in the height of the film thickness is also as a result of flow resistant as a result of 
increase in the surface roughness.

It is noted from Figure 4 that the density of the lubricant increases with increase in pressure. That is because increasing the pressure slightly decreases the volume of the lubricant which leads to an increase in the density. Figure 5 notes that an increase in the surface roughness leads to an increase in pressure. This increase in pressure is a result of the surface roughness increasing the resistance of the lubricant flow hence the pressure rises to maintain the flow continuity. The higher the surface roughness the higher the resistance to the flow of the lubricant hence a rise in the pressure.

The temperature profile of Figure 6 notes that as the load is increased, the temperature of the bearing also increases. This is due to increase in friction in the bearing which results to an increase in temperature. Figure 7 notes that an increase in temperature results to a reduction in the density of the lubricant. This is due to the fact that an increase in temperature leads to increase in the kinetic energy of the molecules of the lubricant which increases its volume. The increase in temperature also leads to a reduction in the viscosity of the lubricant which increase the lubricant's volume. Figure 8 notes that an increase in the surface roughness leads to an increase in temperature. This increase in temperature is due to increase in friction because of the surface roughness.

\section{Conclusion}

In this research, a thermal elastohydrodynamic lubrication of a sliding-rolling bearing with effects of surface roughness, density and load presented. The surface roughness has significance influence on the bearing and lubricant. An increase in the surface roughness increases the pressure and temperature while reduces the film thickness. An increase in load leads to an increase in temperature. Density increases with increase in pressure while reduces with increase in temperature. An increase in density however leads to an increase in film thickness.

\section{Nomenclature}

$\begin{array}{ll}\tau & \text { Shear stress (Pa s) } \\ \tau_{0} & \text { Eyring Shear stress (Pa) } \\ \tau_{m} & \text { Eyring shear stress at mid plate }(\mathrm{Pa}) \\ \gamma & \text { Eyring parameter }(\mathrm{Pa} \mathrm{s}) \\ \eta & \text { The viscosity of the lubricant }(\mathrm{Pa} \mathrm{s}) \\ p_{h} & \text { Maximum Hertzian pressure }(\mathrm{Pa}) \\ \rho & \text { The density of the lubricant }\left(\mathrm{kg} / \mathrm{m}^{3}\right) \\ x & \text { Distance along x direction }(\mathrm{m}) \\ y & \text { Distance along y direction }(\mathrm{m}) \\ u & \text { Velocity along x direction }(\mathrm{m} / \mathrm{s}) \\ u_{m} & \text { mean velocity }(\mathrm{m} / \mathrm{s}) \\ v & \text { Velocity along y direction }(\mathrm{m} / \mathrm{s}) \\ a & \text { Hertzian radius }(\mathrm{m}) \\ t & \text { Time }(\mathrm{s}) \\ h & \text { Film Thickness }(\mathrm{m}) \\ p & \text { Pressure }(\mathrm{Pa}) \\ \mathbb{R} & \text { Dimensionless surface roughness }\end{array}$

$\begin{array}{ll}\mathrm{SR} & \text { Surface roughness }(\mathrm{m}) \\ \mathrm{C}_{\mathrm{p}} & \text { specific heat }(\mathrm{J} / \mathrm{kg} \mathrm{K}) \\ \mathrm{P} & \text { Pressure }(\mathrm{Pa}) \\ \theta & \text { Temperature }(\mathrm{K}) \\ \mathrm{t} & \text { Time }(\mathrm{s}) \\ \mathrm{R} & \text { Radius of rolling element }(\mathrm{m}) \\ \mathrm{Z} & \text { The pressure-viscosity index } \\ E^{\prime} & \text { Elastic modulus }(\mathrm{Pa}) \\ h_{00} & \text { central offset film thickness }(\mathrm{m}) \\ \mathrm{k} & \text { Thermal conductivity }(\mathrm{N} / \mathrm{s} / \mathrm{K}) \\ \mathrm{S} & \text { Viscosity temperature index } \\ i, j & \text { Nodes in } \mathrm{x} \text { and y direction respectively }\end{array}$

\section{Acknowledgements}

Samuel Macharia thanks the Pan African University (PAUSTI) for sponsoring this research paper.

\section{References}

[1] Dowson, D., Higginson, G. R. and Whitaker, A. V. (1962). Elasto-Hydrodynamic Lubrication: A Survey of Isothermal Solutions. J. Mech. Eng. Sci., 4, 121-162.

[2] Gohar, R. and Cameron, A. (1963). Optical Measurement of Oil Film Thickness under Elasto-Hydrodynamic Lubrication. Nature, 200, 458-459.

[3] Lord, J. and Larsson, R. (2001). Effects of slide-roll ratio and lubricant properties on elastohydrodynamic lubrication film thickness and traction. Proc. Inst. Mech. Eng. Part J J. Eng. Tribol., 215, 301-308.

[4] Bair, S. (2005). Shear thinning correction for rolling/sliding elastohydrodynamic film thickness. Proc. Inst. Mech. Eng. Part J J. Eng. Tribol., 219, 69-74.

[5] Kumar, P. and Khonsari, M. M. (2008). EHL circular contact film thickness correction factor for shear-thinning fluids. J. Tribol., 130, 041506-041513.

[6] K. N. Mistry, and M. Priest, "Prediction of the Lubrication Regimes and Friction of Piston Ring Assembly of an I. C Engine Considering the Effect of Surface Roughness," Proceeding of 33rd Leeds-Lyon Symposium on Tribology, 12th-15th September 2006.

[7] Venner, C. H. and Napel, W. E. T. (1992). Surface roughness effects in an EHL line contact. ASME Journal of Tribology, $114,616-622$.

[8] Kumar, P. and Kumar, N. (2014). Surface Roughness Effects in Pure Sliding EHL Line Contacts with Carreau-Type Shear-Thinning Lubricants. Mechanical and Mechatronics Engineering, 8 (6): 311-319.

[9] Bujurke, N. M., Naduvinamani, N. B. and Basti D. P. (2007). Effect of surface roughness on the squeeze film lubrication between curved annular plates. Industrial Lubrication and Tribology, 59 (4): 178-185.

[10] Nadivinamani, N. B., Thasneem, S. F. and Jamal, S. (2010). Effect of roughness on hydrodynamic squeeze films between porous rectangular plates, Tribology International, 43: 2145-2151. 
[11] Kondo, S., Sayles, R. S. and Lowe, M. J. S. (2006). A combined optical-ultrasonic method of establishing the compressibility of high-pressure oil and grease films entrapped in a ball on flat contact. Journal of Tribology, 128 (1): 155-167.

[12] Stahl, J. and Jacobson, O. (2003). Compressibility of lubricants at high pressure. Tribology Transactions, 46 (4): 592-599.

[13] Prasad, D. and Sinha, P. (1988). Non-uniform temperature in non-Newtonian compressible fluid film lubrication of rollers. ASME Journal of Tribology, 110 (4): 653-658.

[14] Moraru, L. and Keith, T. G. (2007). Lobatto point quadrature for thermal lubrication problems involving compressible lubricants: EHL applications. Journal of Tribology, 129 (1): 194-198.

[15] Chu, L., Hsu, H., Lin, J. and Chang, Y. (2009). Inverse approach for calculating temperature in EHL of line contacts. Tribology International, 42 (8): 1154-1162.

[16] Mishra, P. C (2014). Analysis of a Rough Elliptic Bore Journal Bearing using Expectancy Model of Roughness Characterization. Tribology in Industry, 36 (2), 211-219.

[17] Liu, Y., Wang, J., Krupka, I., Hartl, M. and Bair, S. (2008). The shear thinning elastohydrodynamic film thickness of a two-component mixture. Journal of Tribology, 130 (2): 1-7.

[18] Kumar, P., Bair, S., and Khonsari, M. (2008). Full EHL simulations using the actual Ree-Eyring model for shear-thinning lubricants. Journal of Tribology, 131 (1): 1-6.

[19] Shettar, B. M., Hiremath, P. S. and Bujurke, N. M. (2016), Jacobian-free Newton Multigrid method for Elastohydrodynamic line contact with grease as lubricant. International Journal of Computational Engineering Research 6 (8): 2250-3005.

[20] Srikanth, D. V., Kaushal, K. C. and Chenna, K. R. (2012). Determination of a large tilting pad thrust bearing angular stiffness. Tribology International 47: 69-76.

[21] Vishwanath, B. A. and Ashwini, K. (2013). Surface roughness effect on thermohydrodynamic analysis of journal bearings lubricated with couple stress fluids. Nonlinear Engineering, Volume 8, Issue 1: 397-406.

[22] Almqvist, T. and Larsson, R. (2002). The Navier-Stokes approach for thermal EHL line contact solutions. Tribology International, 35: 163-170. 